\title{
Tear N-glycomics in vernal and atopic keratoconjunctivitis
}

\author{
Angela Messina ${ }^{1}$, Angelo Palmigiano ${ }^{2}$, Claudia Tosto ${ }^{2}$, Donata Agata Romeo ${ }^{2}$, Luisa \\ Sturiale $^{2}$, Domenico Garozzo ${ }^{2}$, and Andrea Leonardi ${ }^{3}$ \\ ${ }^{1} \mathrm{CNR}$ \\ ${ }^{2}$ Consiglio Nazionale delle Ricerche (CNR) \\ ${ }^{3}$ University of Padova
}

October 5, 2020

\begin{abstract}
Purpose Tear fluid N-Glycome from patients affected with vernal (VKC) and atopic keratoconjunctivitis (AKC) was investigated to identify specific changes in tears and to recognize possible glyco-biomarkers. Methods The analysis of N-glycans was performed using matrix-assisted laser desorption ionization mass spectrometry on single tear samples. Tears from control normal subjects (CTRL), VKC and AKC patients were processed and treated with peptide N-glycosidase F (PNGase F) to deglycosylate Nglycoproteins. Released N-glycans were purified, permethylated and analyzed by Matrix-Assisted Laser Desorption/IonizationTime Of Flight Mass Spectrometry and tandem Mass Spectrometry (MALDI-TOF MS and MALDI-TOF MS/MS). Results More than 150 complex N-glycans, including highly fucosylated biantennary, triantennary, tetraantennary and bisecting species, were observed in our spectra. Three distinct patterns for CTRL, VKC and AKC patients were identified in terms of relative intensities for some N-glycans structures. Major variations involved bisecting and hyperfucosylated glycoforms. The most intense ions were associated to species at m/z 1907.0 (asialo, agalacto, bisected, biantennary structure - NGA2B) in CTRL MS profiles, at m/z 2605.3 and 2966.5 in VKC, and at m/z 2792.4 in AKC corresponding to a well-known biantennary, disialylated Nglycan. Several peaks were associated to structures bearing one or two Lewis X epitopes. Structures were confirmed by MS/MS analysis. Quantitative differences among the three groups were statistically significant. Conclusions Tear MS profiles are rich in specific glycoforms, particularly those with a high fucosylation degree, indicating both core and peripheral decoration. Tear $\mathrm{N}$-glycome analysis provided important information for a better comprehension of VKC and AKC alterations at the molecular level
\end{abstract}

\section{Purpose}

Tear fluid $N$ - Glycome from patients affected with vernal (VKC) and atopic keratoconjunctivitis (AKC) was investigated to identify specific changes in tears and to recognize possible glyco-biomarkers.

\section{Methods}

The analysis of the $N$ - glycans was performed using matrix-assisted laser desorption ionization mass spectrometry on single tear samples. Tears from control normal subjects (CTRL), VKC and AKC patients were processed and treated with peptide $\mathrm{N}$-glycosidase F (PNGase F) to deglycosylate $N$ - glycoproteins. Released $N$ - glycans were purified, permethylated and analyzed by Matrix-Assisted Laser Desorption/Ionization-Time Of Flight Mass Spectrometry and tandem Mass Spectrometry (MALDI-TOF MS and MALDI-TOF MS/MS).

\section{Results}

More than 150 complex $N$ - glycans, including highly fucosylated biantennary, triantennary, tetraantennary and bisecting species, were observed in our spectra. Three distinct patterns for CTRL, VKC and AKC patients were identified in terms of relative intensities for some $N$ - glycans structures. Major variations involved bisecting and hyperfucosylated glycoforms. 
The most intense ions were associated to species at $\mathrm{m} / z 1907.0$ (asialo, agalacto, bisected, biantennary structure - NGA2B) in CTRL MS profiles, at $m / z 2605.3$ and 2966.5 in VKC, and at $m / z 2792.4$ in AKC corresponding to a well-known biantennary, disialylated $N$ - glycan. Several peaks were associated to structures bearing one or two Lewis X epitopes. Structures were confirmed by MS/MS analysis. Quantitative differences among the three groups were statistically significant.

\section{Conclusions}

Tear MS profiles are rich in specific glycoforms, particularly those with a high fucosylation degree, indicating both core and peripheral decoration. Tear $N$ - glycome analysis provided important information for a better comprehension of VKC and AKC alterations at the molecular level.

Keywords: Glycomics, vernal keratocojunctivitis, atopic keratoconjunctivitis, tears, mass spectrometry

\section{INTRODUCTION}

The ocular tear film is a complex mixture of ions, small molecules, glycoproteins and hundreds of proteins, many of which are bioactive $[1,2]$. The functions of the tear film consist of covering and wetting the surface of the cornea and the conjunctiva, maintaining their integrity, protecting against microbial challenge and preserving the visual acuity. A number of studies reported on differential proteomic analysis of tear fluids of healthy subjects and patients as an approach successfully employed for biomarker search and identification in dry eye disease [3,4], in climatic droplet keratopathy [5], in glaucoma [6] and in vernal keratoconjunctivitis (VKC) [7]. More recently, an increasingly growing interest in glycomic studies has emerged, since qualitative and quantitative glycan alterations are often crucial to afford key information on pathological mechanisms in a large variety of disorders and severe diseases such as rheumatoid arthritis [8,9], diabetes [10], inflammation [11], tumors [12] and Alzheimer's disease [13-19].

Glycosylation is a complex post-translational modification of proteins, very sensitive to the biochemical environment, depending on the action of several glyco-enzymes. Even though it is not under direct genetic control, glycosylation affects several biological processes [20,21].

Glycosylation is an important factor in regulating the ocular surface homeostasis [22]. It was reported that inflammatory stress causes $N$-glycan processing deficiency in ocular autoimmune disease [23], while tear fluid $N$ - glycosylation can provide specific and sensitive methods for biomarker discovery and disease diagnosis $[24,10]$. These pioneering works on tear glycomics suggested a reduced $N$-glycans fucosylation degree as potential biomarker for rosacea [24], and significant quantitative changes in some $N$ - glycans in diabetes patients compared to diabetic retinopathy [10]. Both studies reported the identification of about $50 \mathrm{~N}$ glycan species, pinpointing structures containing up to seven fucoses by Matrix-Assisted Laser Desorption Ionization Time of Flight Mass Spectrometry (MALDI TOF MS) [24], and complex $N$-glycans carrying up two sialic acids detected by Electrospray Mass Spectrometry (ESI-MS) [10]. Despite these achievements, a complete $N$ - glycans profile of tear fluid is still missing because such investigations were performed on free (underivatized) glycans, undergoing sialic acids loss and fucose fragmentation when analyzed by MALDI MS and ESI MS, respectively. Nevertheless, such instances did not affect the aforesaid outcomes, as the analyses by MALDI MS dealt with the identification of $N$ - glycan hyperfucosylated structures, while the research handled by ESI MS mainly pointed to extend the characterization of $N$ - and $O$-linked sialylated species.

To acquire the human tear fluid $N$ - glycoprofile without any loss of information, we carried out MALDI MS analysis on permethylated $N$ - glycans, a widely known glycomic approach allowing a high-sensitivity analysis of oligosaccharide species avoiding glycosidic linkage fragmentation. In the present study we moreover applied the MALDI MS and MS/MS approach to deeply characterize and compare the $N$ - linked glycan profiles of tear fluid from normal subjects and from patients affected by VKC and atopic keratoconjunctivitis (AKC), aiming at identifying changes and, possibly, specific glycan features (glyco-biomarkers) associated to these ocular diseases.

VKC and AKC are two severe and persistent types of ocular allergy affecting the conjunctiva and the cornea, impairing the physiology and the function of the ocular surface and possibly causing significant complications 
leading to vision loss [25]. VKC is a chronic bilateral conjunctival inflammation typically occurring in areas with tropical and temperate climates and with a significant male preponderance, affecting mostly children and young adults [26]. AKC is a bilateral conjunctival and eyelid inflammation, which should be considered the ocular localization of atopic dermatitis [27]. Both VKC and AKC have a characteristic ropey, stringy mucous and/or serous discharge, and possible corneal complications, such as superficial punctate keratopathy (SPK), macroerosions and ulcers. If inappropriately treated, both VKC and AKC can lead to severe complications such as glaucoma, corneal scarring, and blindness. To date, no specific laboratory tests are suitable for VKC and AKC diagnosis and monitoring.

\section{METHODS}

\section{Subjects and tear collection}

The research followed the Tenets of the Declaration of Helsinki. Institutional Review Board approval was obtained. Written informed consent was obtained from all subjects or, in the case of minors, from their parents after explanation of the nature and the possible consequences of the study. Eleven normal control subjects (CTRL) (5 females, 6 males; mean age 19+5.3; range 7-30), 23 active VKC patients (mean age $15+10.4$; range 5-40) and 7 active AKC patients (mean age 16+4.1; range 8-20) were included in the study (supplementary Table 1). All patients and CTRL were free of medication for at least 5 days before sample collection. Tears were collected gently from the external canthus in the morning between 9am and 11am using a glass capillary tube. Tear samples were centrifuged, supernatants were collected and immediately frozen at $-80^{\circ} \mathrm{C}$ before analyzed. Samples were not pooled from both eyes or from different patients.

\section{Sample preparation}

$8 \mu \mathrm{L}$ of tears from CTRL, VKC and AKC patients were diluted $1 / 10$ in a $0.1 \%$ denaturant solution of RapiGest (Waters Corporation, Milford, Massachusetts) in $50 \mathrm{mM} \mathrm{NH}_{4} \mathrm{HCO}_{3}$. Denaturated (glyco)proteins were reduced by adding $100 \mathrm{mM}$ dithiothreitol (DTT) in $50 \mathrm{mM} \mathrm{NH}_{4} \mathrm{HCO}_{3}$ (final concentration $5 \mathrm{mM}$ ) and incubated for $30 \mathrm{~min}$ at $56^{\circ} \mathrm{C}$. Subsequently, cysteine alkylation was achieved by an addition of $100 \mathrm{mM}$ iodoacetic acid (IAA) in $50 \mathrm{mM} \mathrm{NH}_{4} \mathrm{HCO}_{3}$ (final concentration $15 \mathrm{mM}$ ) and incubation for 40 min at room temperature in the dark. The $N$ - linked glycans were enzymatically released by the action of peptide-Nglycosidase F (PNGase F, from Flavobacterium meningosepticum EC 3.5.1.52; Roche Diagnostics GmbH) ( $2 \mathrm{ml}$ corresponding to 2 units) at $37^{\circ} \mathrm{C}$ overnight and then purified by solid-phase extraction using Hypercarb cartridges (Thermo Fisher Scientific, Bellefonte, USA). PNGase F treatment is higly efficient in these conditions [28].

Glycan permethylation was afforded as previously described [29] according to Ciucanu and Kerek protocol [30], to further enhance detection sensitivity upon MS investigation. Permethylated $N$ - glycans were then subjected to MS investigation by MALDI TOF MS and MALDI TOF/TOF MS/MS to achieve a detailed structural elucidation of some $N$ - glycans and univocally establish their structures.

\section{MALDI TOF MS and MS/MS analysis}

A few microliters of permethylated $\mathrm{N}$-glycan samples, resuspended in $\mathrm{MeOH}$, were mixed with the same volume of matrix solution (10 mg/ml 5-Chloro-2-mercaptobenzothiazole, CMBT in $\mathrm{MeOH} / \mathrm{H}_{2} \mathrm{O}$ 80/20). MALDI TOF and MALDI TOF/TOF mass spectra were recorded in reflectron positive mode using a 4800 MALDI TOF/TOF (Applied Biosystems) instrument, equipped with an Nd:YAG laser at 355-nm and $200 \mathrm{~Hz}$ repetition rate. In MS mode, 1200 shots were accumulated for each spectrum, with a resolution greater than $15 \mathrm{~K}$ and a mass accuracy better than $80 \mathrm{ppm}$. A 4700-calibration standard kit, calmix (Applied Biosystems) was used as external calibrant for the MS mode, and [Glu1] fibrinopeptide B human (Sigma) was used as external calibrant for the MS/MS mode $(1 \mu \mathrm{L}$ of TOF/TOF Calibration Mixture in $24 \mu \mathrm{L}$ of CHCA matrix solution).

The MS data were processed by DataExplorer 4.9 software and the N-glycan species were identified by bioinformatics tools, such as GlycoMod (http://web.expasy.org/glycomod/), Glycoworkbench v2.1 [31] and by tools provided by the Consortium for Functional Glycomics (CFG). 


\section{Tear serotransferrin quantitative analysis}

To validate the glycomic results, tears from the $7 \mathrm{AKC}$ and 7 VKC patients, and from 7 CTRL were used to measure tear concentrations of serotransferrin by the commercial quantitative immunochemical analysis (Dimension Vista System, Siemens, Erlangen, Germany) (Limit of Detection (LOD) is $0.002 \mathrm{mg} / \mathrm{L}$ ).

\section{Statistical analyses}

Statistical analyses were accomplished using a commercially available software program (PASW Statistics 18). Specificity and relevance of quantitative differentiations were established by ANOVA p-values and results were expressed as mean values $\pm \mathrm{SD}$ (standard deviation). In relation to serotransferrin tear levels, since none of the groups showed a normal distribution (Shapiro-Wilk's test $\mathrm{p}<0.05$ ), sample values were expressed by median \pm interquartile range (IRQ). Comparison among groups was performed by U Mann-Whitney test with normal approximation and 0.5 continuity correction. For statistical significance, the threshold p value was $\mathrm{p}<0.05$.

\section{RESULTS}

\section{$N$-glycosylation analysis of control tear fluid}

Tear samples from the 11 CTRL were processed as described and analyzed by MALDI TOF MS in order to define the typical tear $N$ - glycan pattern in healthy subjects. Permethylated glycans were detected in positive polarity as $\mathrm{Na}^{+}$ion adducts, whose molecular masses differ from their theoretical protonated counterpart by $22 \mathrm{u}$ (atomic mass unit). All the CTRL mass spectra were found very similar to the profile in Figure 1A, showing more than 150 peaks corresponding to $[\mathrm{M}+\mathrm{Na}]^{+}$pseudo-molecular ions from $\mathrm{m} / z 1579.8$ to $\mathrm{m} / z$ 4432.2 (supplementary Table 2). Spectra showed the occurrence of a wide range of $N$ - glycans categories including oligomannose, complex bi-, tri-, and tetra-antennary, neutral, acidic, bisected and hybrid species. Additionally, the main peaks were further analyzed by MALDI TOF/TOF MS/MS. This analysis was crucial to discriminate, in most cases, between isomeric glycoforms or to confirm the presence of two or even more isomeric glycoforms associated to a single molecular mass.

Oligomannose $N$-glycans

Peaks related to oligomannose glycans were observed in Figure 1A atm/z 1579.8 (Man5), 1783.9 (Man6), 1988.0 (Man7), 2192.1 (Man8). The MS/MS spectra (supplementary Figures S1, S2, S3, S4), confirmed these assignments.

Mono-, di- and multi-fucosylated $N$-glycans

Amongst the $N$ - glycan structures (approximately 150) listed in supplementary Table 2, 116 (about 75\%) were fucosylated, being 41 out 116 mono-fucosylated and 75 out 116 bearing more than one fucose unit, with two glycans having up to seven fucose moieties. Core fucosylated and peripheral fucosylated $N$ - glycans, depending on fucose localization, were both present in the CTRL tear $N$ - glycome profile. In the present study, core fucosylation (due to an $\alpha 1-6$ fucose linked to the $N$ - acetylglucosamine bound to the protein), and peripheral fucosylation, (due to a fucose moiety 1-3 or 1-4 linked to an antennary N-acetylglucosamine or 1-2 linked to a galatose [32]) were characterized by the occurrence of specific MS/MS ions. Antennary fucosylation allowed us to recognize specific epitopes belonging to the Lewis family (structural features typical of blood group antigens [33]) (see supplementary Table 3).

Corefucosylated glycans and/or $N$ - glycans bearing $\mathrm{Le}^{\mathrm{x} / \mathrm{a}}, \mathrm{sLe}^{\mathrm{x} / \mathrm{a}}$ or $\mathrm{Le}^{\mathrm{y} / \mathrm{b}}$ motifs were identified (current approaches based MALDI MS and MS/MS on permethylated $N$ - glycans do not provide informations on linkage positions, in order to discriminate $(\mathrm{s}) \mathrm{Le}^{\mathrm{a}}$ from $(\mathrm{s}) \mathrm{Le}^{\mathrm{x}}$ and $\mathrm{Le}^{\mathrm{b}}$ from $\mathrm{Le}^{\mathrm{y}}$ ).

The occurrence of a Le $\mathrm{e}^{\mathrm{x} / \mathrm{a}}$ epitope was confirmed by the ion at $m / z 660.3$ in the fragmentation spectra of the glycan strucures at $m / z 2418.2,2592.3,2605.3,2766.4$ and 2779.4. Figure 2 shows the MS/MS spectrum of the latter peak, whereas the other fragmentation spectra are reported in the supplementary (supplementary Figures S5, S6, S7, S8). MS/MS analysis allowed us to identify some ions corresponding to more than one 
isomeric structure (i.e., peak at $m / z 2605.3$ in Figure $\mathrm{S} 7$ is actually related to a mixture of two glycans, a corefucosylated one and a second bearing a $\mathrm{Le}^{\mathrm{x} / \mathrm{a}}$ epitope).

The $\mathrm{sLe}^{\mathrm{x} / \mathrm{a}}$ motif, recognized through the fragment at $m / z 1021.5$, was associated to the glycan structures at $m / z 3127.6,3140.6$ and 3589.8 (supplementary Figures S9, S10, S11). Interstingly, the structure at $\mathrm{m} / z$ 3127.6 corresponded to a glycoform owning four fucoses, suggesting that corefucose, sLe ${ }^{\mathrm{x} / \mathrm{a}}$ and $\mathrm{Le}^{\mathrm{y} / \mathrm{b}}$ epitopes are all present in the same glycan (supplementary Figure 9).

$\mathrm{Le}^{\mathrm{y}}$ (and/or Le ${ }^{\mathrm{b}}$ ) pattern is proven by the fragment at $m / z 834.4$ occurring in the fragmentation spectra of the glycans at $m / z 2418.2,2592.3,2766.4,2940.5$ and 3127.6 (see supplementary Figures S5, S6, S8, S12 and $\mathrm{S} 9$ respectively).

\section{Bisected N-glycans}

A number of complex structures with bisecting GlcNAc were present in CTRL tear fluid profiles, including two very intense ions at $m / z 1906.9$ and 2081.1 that correspond to the agalactosyl- biantennary structure A2B and to the same structure core-fucosylated (FA2B), respectively. Since permethylated bisected species showed no characteristic fragments by MALDI MS/MS, structural characterization was achieved by comparing the fragmentation spectra with the model compound as already reported [13].

\section{Sialylated $N$-glycans}

A large portion of the identified tears $N$ - glycans beared a sialic acid as terminal antennary residue, with the two typical diagnostic fragments observed by MS/MS at $m / z 847.4$ and at $m / z 1021.5$, corresponding to the sialylated lactosamine epitope, and to fucosylated homologous (sLe ${ }^{\mathrm{x} / \mathrm{a}}$ motif), respectively.

\section{Hybrid glycans}

A minor component of hybrid glycans was also detected. MS/MS analysis confirmed that peaks at $m / z 2156.1$ (supplementary Figure S13) and 2360.2 (supplementary Figure S14) corresponded to hybrid structures with terminal sialic acid and core fucosylation.

\section{Tears $N$-glycosylation of VKC patients}

MALDI -TOF $N$ - glycans analyses were performed in $23 \mathrm{VKC}$ individual tear samples. A unique distinctive profile emerged from these patients (Figure 1B). Peaks at $\mathrm{m} / z 2605.3$ and 2966.5 corresponded to biantennary monofucosylated structures bearing one and two sialic acids respectively, whereas the ions at $\mathrm{m} / z 3415.7$ and 3776.9 matched monofucosylated triantennary structures bearing two and three sialic acids respectively. These two structures showed the fucose unit linked either to the chitobiose core and to the antennary $N$ acetylglucosamine.

Interstingly, the ion at $m / z 2792.4$, related to the biantennary disialo structure, was more intense in VKC patiens compared to CTRL (Figure 1A). On the contrary, peaks corresponding to truncated $N$ - glycans at $\mathrm{m} / z$ 1907.0, 2081.0 and 2285.1 were two- or three-fold less intense than the corresponding ones in the CTRL. Statistical differences between VKC and CTRL in relative intensities of the peaks are shown in Figure 3a. Interestingly, in VKC glyco-profiles the ions corresponding to the sialylated structures are more abundant, whereas, bisected $N$ - glycan series is less abundant.

\section{Tear $N$-glycosylation of AKC patients}

All tears samples from the 7 AKC patients showed similar glyco-profiles (Figure 1C). Compared to CTRL and VKC, AKC spectra showed a strong increase of the species at $m / z 2431.2$ and 2792.4 (corresponding to biantennary $N$ - glycans carrying one and two sialic acids respectively) and also a decrease of the ions at $\mathrm{m} / \mathrm{z} 2605.3$ and m/z 2966.5 associated to their fucosylated counterparts. Minor increase of the $N$-glycans corresponding to the sialylated triantennary series $(\mathrm{m} / z 3602.8$ and $\mathrm{m} / z$ 3776.9) was detected. Furthermore, significant increase of oligomannose and bisected species besides a decrease of sialylated species, with the exception of the peak atm/z 2953.5, were observed (Figure 3B). 


\section{Tear serotransferrin quantitative analysis}

Quantitative analysis of serotransferrin, associated to the peak at $m / z 2792.4$, confirmed the significant increase of this protein in AKC and VKC tears compared to CTRL $(\mathrm{p}=0.0014)$ and higher levels in AKC compared to VKC ( $\mathrm{p}=0.0039)$ (Figure 4$)$.

\section{DISCUSSION}

MALDI-MS profiling and MALDI-MS/MS characterization of permethylated $N$ - glycans permitted to extend the repertoire of the identified $N$ - glycans in the normal control tear fluid up to [?] 150, as reported in Table S2. Many of the glycans shown in this table were detected for the first time because, as already mentioned in the introduction, MALDI analysis of not-derivatized glycans underestimates the amount of sialylated glycans, and ESI-MS underestimates the occurrence of strucures decorated with fucose units, whereas MALDI MS of permethylated glycans is an effective method to detect both these $N$-glycan series. Although this approach does not allow to trace the proteins from which the identified glycans originated, it is important to point out that two glycoproteins are much more abundant in the normal tear fluid than others [34]: lactoferrin $(1.56 \mathrm{~g} / \mathrm{L})$ and secretory $\operatorname{IgA}(2.07 \mathrm{~g} / \mathrm{L})$. Another major glycoprotein certainly contributing to the overall glycosylation profile is Immunoglobulin $\mathrm{G}(\mathrm{IgG})$, which is present, however, in a much lower concentration $(0.004 \mathrm{~g} / \mathrm{L})[34]$. The $N$ - glycosylation of lactoferrin and IgA has been extensively studied $[35,36]$ and it can be supposed that oligomannose and asymmetrically branched $N$-glycans originate largely from lactoferrin, complex-type glycans (mainly biantennary and bisected) derive predominantly by IgA, multi-fucosylated glycans come from the Secretory Component of IgA, and monoantennary and hybrid-type glycans arise from the joining chain of secretory IgA.

All the obtained tear MS profiles were rich in specific glycoforms, particularly those with a high fucosylation degree, indicating both core and peripheral decoration, these last essential component of antigens belonging to the Lewis family on cell membrane surface. Increased levels of fucosylation have been implicated in several diseases: a) inflammatory diseases, as $N$-glycans fucosylation plays a regulatory role for selectin-dependent leukocyte adhesion [37]; b) immunological response, owing to the regulatory role of fucosylated glycoforms in immune response and to the autoimmunity development [38,39] and c) cancer, promoting TNF- $\alpha$ activity in M1 inflammatory macrophages [40].

Large differences emerged from the comparison of $N$ - glycome profiles from control, VKC and AKC tear fluids. In control samples, the base peak was the bisected species at $m / z$ 1907.0. In VKC, dominant ions were assigned to the biantennary and core-fucosylated glycans at $m / z 2605.3$ and $m / z 2966.5$. The AKC MS profiles showed a huge increase of the ion at $m / z$ 2792.4, (biantennary, disialylated unfucosylated glycoform), with respect to and VKC tears. These glycomic data were in good agreement with our previous results on quantitative proteomics of VKC tear fluid, showing increase of serotransferrin and lactotransferrin with a concomitant reduction of immunoglobulins [7]. Altoghether, these results led to assume that the peaks with increased intensities refer to glycans from lactotransferrin $(\mathrm{m} / z 2966.5$ and 2605.3) [36,41,42] and from serotransferrin $(m / z$ 2792.4) [29,43], whereas peaks showing decreased intensity are associated to immunoglobulins.

Serotransferrin is an iron-binding plasma glycoprotein, primarily expressed in the liver but also in corneal epithelial cells [44]. It has been shown that patients with allergy and allergic keratoconjunctivitis suffer an increased oxidative stress condition $[45,46]$. The capacity of binding free iron to protect against toxic radical oxygen species, gives to the serotransferrin an antioxidant effects inhibiting iron-mediated oxidation [45]. Serotransferrin is usually present in tears in very low concentrations, belongs to the ocular innate immune system and contributes, with lactoferrin, to the iron-sequestration mechanisms active against pathogens.

Serotransferrin and other serum proteins like albumin and IgG are not typically present in the ocular surface at high levels and, presumably, they may derive from plasma through a passive filtration from vessels. Their presence in the tear film could be due to a serum leakage, in response to ocular inflammation, to a stimulation of the conjunctiva or to a mild ocular trauma [47]. However, the dramatic increase of species at $\mathrm{m} / z 2792.4$ in VKC and AKC MS profiles, the high serotransferrin tear levels in AKC and, in lesser extend, in VKC 
and the relative lower peaks related to immunoglobulins, may suggest a local production of serotransferrin in addition of serum leakage due to the ocular inflammation. A reduction in serotransferrin tear levels has been shown in a rabbit model of Sjögren syndrome-associated dry eye [48] and in saporin toxin denervated lacrimal glands rat-model despite normal tear production [49]. Differently from dry eye, ocular allergy is characterized by increased tearing and corneal sensitivity and by corneal nerve fiber abnormalities [50,51]. The antimicrobial, antiviral, antiparasitic and anti-inflammatory activity of serotransferrin and of other overexpressed tear fluid proteins, may explain the low rate of infection in ocular allergy patients and may function as an up-regulating mechanism to down-regulate the effects of pro-inflammatory cytokine overexpressed by the allergic reaction [52].

In conclusion, we identified in normal control tears multiple specific glycoforms, many of whom with a high fucosylation degree. Tear $N$-glycome analysis highlighted profound and specific changes of tear proteome in allergic keratoconjunctivitis compared to control tears.

\section{Funding}

This work was partially supported by a generous donation from the "Stella Lucente" trust

\section{Disclosure/Conflict of Interest statement}

The authors declare no conflicts of interest

\section{Figure legends}

Fig. 1. MALDI TOF mass spectra, with major assignments, of permethylated $N$ - glycans released by PNGase F from control (CTRL) (A), vernal keratoconjunctivitis (VKC) (B) and atopic keratoconjunctivitis (AKC) (C) tears.

$N$ - acetylglucosamine (GlcNAc): blue square; Mannose (Man): green circle; Galactose (Gal): yellow circle; Sialic acid (NeuAc): purple lozenge; Fucose (Fuc): red triangle.

Fig. 2. MALDI TOF/TOF fragmentation analysis of the parent ion at $m / z 2779.4$ present in the MS spectra from a control tear.

$N$ - acetylglucosamine (GlcNAc): blue square; Mannose (Man): green circle; Galactose (Gal): yellow circle; Sialic acid (NeuAc): purple lozenge; Fucose (Fuc): red triangle.

Fig. 3. Comparison of statistically significant relative intensities between control subjects (CTRL) and vernal keratoconjunctivitis (VKC) patients (a), CTRL and atopic keratoconjunctivitis (AKC) patients (b). Most intense 30 peaks were compared and only statistically significant are shown. Bars indicate standard errors $\left({ }^{*} \mathrm{p}<0.01 ;{ }^{*} \mathrm{p}<0.001\right)$.

Fig. 4. Tear levels of serotransferrin, by commercial immunometric analysis, in the cohort of atopic keratoconjunctivitis (AKC) and vernal keratoconjunctivitis (VKC) patients and control subjects (CTRL). Tear levels were significanlty higher in AKC and VKC compared to CTRL, and in AKC compared to VKC (p-values by U Mann-Whitney test).

\section{Supplementary Materials}

Supplementary Table 1. Clinical data from VKC and AKC patients

Supplementary Table 2. $N$ - glycans identified in human tears

Supplementary Table 3. Structures of Lewis determinants

Supplementary figure Legends

Figures S1-S14: MALDI-TOF/TOF fragmentation analysis of the parent ion at $\mathrm{m} / z 1579.8, \mathrm{~m} / z \mathrm{z} 1783.9, \mathrm{~m} / \mathrm{z}$ 1988.0, 2192.1, $\mathrm{m} / z$ 2418.2, $\mathrm{m} / z$ 2592.3, $\mathrm{m} / z$ 2605.3, $\mathrm{m} / z 2766.4, \mathrm{~m} / z 3127.6, \mathrm{~m} / z$ 3140.6, $\mathrm{m} / \mathrm{z} 3589.8, \mathrm{~m} / \mathrm{z}$ 
2940.5, $\mathrm{m} / z$ 2156.1, $\mathrm{m} / z 2360.2$ respectively, present in the mass spectrum from a CTRL tear. MS/MS spectra recorded in several samples including VKC and AKC showed no differences.

$N$ - acetylglucosamine (GlcNAc): blue square; Mannose (Man): green circle; Galactose (Gal): yellow circle; Sialic acid (NeuAc): purple lozenge; Fucose (Fuc): red triangle.

Acknowledgments.

a. Funding/Support: This work was partially supported by a generous donation from the "Stella Lucente" trust

b. Conflicts of Interest:

Angela Messina: No Conflicts of Interest

Angelo Palmigiano: No Conflicts of Interest

Claudia Tosto: No Conflicts of Interest

Donata Agata Romeo: No Conflicts of Interest

Luisa Sturiale: No Conflicts of Interest

Domenico Garozzo: No Conflicts of Interest

Andrea Leonardi: No Conflicts of Interest.

c. Contributions to Authors in each of these areas: Angela Messina (AM), Angelo Palmigiano (AP), Claudia Tosto (CT), Donata Agata Romeo (DAR), Luisa Sturiale (LS), Domenico Garozzo (DG), Andrea Leonardi $(\mathrm{AL})$

Conseption and Design: AL, DG, AM

Analysis and interpretation: DG, AL, AM, LS, AP

Writing the article: AM, AL, DG, LS

Critical revision of the article: LS, DAR, CT

Final approval of the article: AL, SD, JLF, LD, SD, DR, MK

Data Collection: AM, AP, CT, DAR, LS, DG, AL

Provision of materials, patients, or resources: AL, DG

Statistical expertise: LS, CT

Obtaining funding: DG

Literature search: AM, AL, DG, LS,

Administrative, technical or logistic support: AL, DG, AP

d. Statement about Conformity with Author Information: none

e. Other Acknowledgments: none

\section{References}

1. Zhou L, Zhao SZ, Koh SK, Chen L, Vaz C, Tanavde V et al. In-depth analysis of the human tear proteome. J Proteomics 2012;75:3877-85.

2. de Souza GA, Godoy LM, Mann M. Identification of 491 proteins in the tear fluid proteome reveals a large number of proteases and protease inhibitors. Genome Biol 2006;7:R72. 
3. Zhou L, Beuerman RW, Chan CM, Zhao SZ, Li XR, Yang H et al. Identification of tear fluid biomarkers in dry eye syndrome using iTRAQ quantitative proteomics. J Proteome Res 2009;8:4889-905.

4. Tong L, Zhou L, Beuerman RW, Zhao SZ, Li XR. Association of tear proteins with Meibomian gland disease and dry eye symptoms. Brit J Ophthalmol 2011;95(6):848-52.

5. Lei Z, Beuerman RW, Chew AP, Koh SK, Cafaro TA, Urrets-Zavalia EA et al. Quantitative analysis of $N$ - linked glycoproteins in tear fluid of climatic droplet keratopathy by glycopeptide capture and iTRAQ. J Proteome Res 2009;8:1992-2003.

6. Wong TT, Zhou L, Li J, Tong L, Zhao SZ, Li XR, et al. Proteomic profiling of inflammatory signaling molecules in the tears of patients on chronic glaucoma medication. Invest Ophth Vis Sci 2011;52(10):7385-91.

7. Leonardi A, Palmigiano A, Mazzola EA, Messina A, Milazzo EMS, Bortolotti M et al. Identification of human tear fluid biomarkers in vernal keratoconjunctivitis using iTRAQ quantitative proteomics. Allergy 2014;69(2):254-56.

8. Parekh RB, Dwek RA, Sutton BJ, Fernandes DL, Leung A, Stanworth D et al. Association of rheumatoid arthritis and primary osteoarthritis with changes in the glycosylation pattern of total serum IgG. Nature 1985;316(6027):452-57.

9. Parekh R, Roitt I, Isenberg D, Dwek R, Rademacher T. Age-related galactosylation of the $N$ - linked oligosaccharides of human serum IgG. J Exp Med 1988;167(5):1731-36.

10. Nguyen-Khuong T, Everest-Dass AV, Kautto L, Zhao Z, Willcox MD, Packer NH. Glycomic characterization of basal tears and changes with diabetes and diabetic retinopathy. Glycobiology 2015;25(3):269-83.

11. Wang J, Yang D, Li C, Shang S, Xiang J. Expression of extracellular matrix metalloproteinase inducer glycosylation and caveolin-1 in healthy and inflamed human gingiva. J Periodontal Res 2014;49(2):197-204.

12. Adamczyk B, Tharmalingam T, Rudd PM. Glycans as cancer biomarkers. Biochim Biophys Acta(BBA)Gen Subj 2012;1820(9):1347-53.

13. Palmigiano A, Barone R, Sturiale L, Sanfilippo C, Bua RO, Romeo DA et al. CSF $N$ - glycoproteomics for early diagnosis in Alzheimer's disease. J Proteomics 2016;131:29-37.

14. Palmigiano A, Messina A, Bua RO, Barone R, Sturiale L, Zappia M et al.: CSF $N$ - Glycomics using MALDI MS techniques in Alzheimer's Disease, in Perneczky R (ed): Biomarkers for Alzheimer's Disease Drug Development. New York, NY: Humana Press, 2018, pp. 75-91.

15. Messina A, Palmigiano A, Bua RO, Romeo DA, Barone R, Sturiale L et al.: CSF N-Glycoproteomics Using MALDI MS Techniques in Neurodegenerative Diseases, in Santamaria E, Fernandez-Irigoyen J (eds): Cerebrospinal Fluid (CSF) Proteomics. New York, NY: Humana, 2019, pp. 255-72.

16. Kizuka Y, Kitazume S,Taniguchi N. N-glycan and Alzheimer's disease. Biochim Biophy Acta (BBA)General Subjects 2017;1861(10):2447-54.

17. Cho BG, Veillon L, Mechref Y.N- Glycan Profile of Cerebrospinal Fluids from Alzheimer's Disease Patients Using Liquid Chromatography with Mass Spectrometry. J Proteome Res 2019;18(10):3770-79.

18. Quaranta A, Karlsson I, Ndreu L, Marini F, Ingelsson M, Thorsen G. Glycosylation profiling of selected proteins in cerebrospinal fluid from Alzheimer's disease and healthy subjects. Anal Methods-UK 2019;11(26):3331-40.

19. Schedin-Weiss S, Gaunitz S, Sui P, Chen Q, Haslam SM, Blennow K, et al. Glycan biomarkers for Alzheimer disease correlate with T-tau and P-tau in cerebrospinal fluid in subjective cognitive impairment. FEBS J 2019.

20. Dwek RA. Glycobiology: toward understanding the function of sugars. Chem Rev 1996;96(2):683-720. 
21. Kobata A. A journey to the world of glycobiology. Glycoconjugate J 2000;17(7-9):443-64.

22. Rodriguez Benavente MC, Argueso P. Glycosylation pathways at the ocular surface. Biochem Soc T 2018;46(2):343-50.

23. Woodward AM, Lehoux S, Mantelli F, Di Zazzo A, Brockhausen I, Bonini S, Argueso P. Inflammatory stress causes N-glycan processing deficiency in ocular autoimmune disease. Am J Pathol 2019;189(2):283-94.

24. Vieira AC, An HJ, Ozcan S, Kim JH, Lebrilla CB, Mannis MJ. Glycomic analysis of tear and saliva in ocular rosacea patients: the search for a biomarker. Ocul Surf 2012;10(3):184-92.

25. Leonardi A, Doan S, Fauquert JL, Bozkurt B, Allegri P, Marmouz F et al. Diagnostic tools in ocular allergy. Allergy 2017;72(10):1485-98.

26. Leonardi A. Vernal keratoconjunctivitis: pathogenesis and treatment. Prog Retin Eye Res 2002;21:31939.

27. Hu Y, Matsumoto Y, Dogru M, Okada N, Igarashi A, Fukagawa K et al. The differences of tear function and ocular surface findings in patients with atopic keratoconjunctivitis and vernal keratoconjunctivitis. Allergy 2007;62(8):917-25.

28. Yu YQ, Gilar M, Kaska J, Gebler JC. A rapid sample preparation method for mass spectrometric characterization of N-linked glycans. Rapid Comm Mass Sp 2005;19(16):2331-36.

29. Sturiale L, Barone R, Garozzo D. The impact of mass spectrometry in the diagnosis of congenital disorders of glycosylation. J Inherit Metab Dis 2011;34:891-99.

30. Ciucanu I, Kerek F. A simple and rapid method for the permethylation of carbohydrates. Carbohyd Res 1984;131:209-17.

31. Ceroni A, Maass K, Geyer H, Geyer R, Dell A. Haslam SM. GlycoWorkbench: a tool for the computerassisted annotation of mass spectra of glycans. J Proteome Res 2008;7:1650-59.

32. Ma B, Simala-Grant JL, Taylor DE. Fucosylation in prokaryotes and eukaryotes. Glycobiology 2006;16(12):158R-184R.

33. Stanley P, Cummings RD: Structures Common to Different Glycans, in: Varki A, Cummings RD, Esko JD et al (eds): Essentials of Glycobiology 3rd edition. Cold Spring Harbor (NY): Cold Spring Harbor Laboratory Press, 2017, pp 161-78.

34. Tiffany JM: Tears and conjunctiva, in Harding JJ (ed.): Biochemistry of the eye. London, Chapman \& Hall Medical, 1997, pp. 45-78.

35. Plomp R, de Haan N, Bondt A, Murli J, Dotz V, Wuhrer M. Comparative glycomics of immunoglobulin A and G from saliva and plasma reveals biomarker potential. Front Immunol 2018;9:2436.

36. Karav S, German JB, Rouquie C, Le Parc A, Barile D. Studying lactoferrin $N$-glycosylation. Int J Mol Sci 2017;18(4):870.

37. Li J, Hsu HC, Mountz JD, Allen JG. Unmasking Fucosylation: from Cell Adhesion to Immune System Regulation and Diseases. Cell Chem Biol 2018;25(5):499-512.

38. Bianco GA, Toscano MA, Ilarregui JM, Rabinovich GA. Impact of protein-glycan interactions in the regulation of autoimmunity and chronic inflammation. Autoimmun Rev 2006;5(5):349-56.

39. Lowe JB. Glycan-dependent leukocyte adhesion and recruitment in inflammation. Curr Opin Cell Biol 2003;15(5):531-38.

40. Li J, Hsu HC, Ding Y, Li H, Wu Q, Yang P et al. Inhibition of fucosylation reshapes inflammatory macrophages and suppresses type II collagen-induced arthritis. Arthritis Rheumatol 2014;66(9):2368-79. 
41. Barboza M, Pinzon J, Wickramasinghe S, Froehlich JW, Moeller I, Smilowitz JT et al. Glycosylation of human milk lactoferrin exhibits dynamic changes during early lactation enhancing its role in pathogenic bacteria-host interactions. Mol Cell Proteomics 2012;11(6):p. mcp.M111.015248.

42. Le Parc A, Dallas D, Duaut S, Leonil J, Martin P, Barile D. Characterization of goat milk lactoferrin $N$ glycans and comparison with the $N$ - glycomes of human and bovine milk. Electrophoresis 2014;35(11):156070 .

43. Sturiale L, Barone R, Palmigiano A, Ndosimao CN, Briones P, Adamowicz M et al. Multiplexed glycoproteomic analysis of glycosylation disorders by sequential yolk immunoglobulins immunoseparation and MALDI-TOF MS. Proteomics 2008;8(18):3822-32.

44. Kompella UB, Sundaram S, Raghava S, Escobar ER. Luteinizing hormone-releasing hormone agonist and transferrin functionalizations enhance nanoparticle delivery in a novel bovine ex vivo eye model. Mol Vis $2006 ; 12: 1185-98$.

45. Bakkeheim E, Mowinckel P, Carlsen KH, Burney P, Carlsen KC. Altered oxidative state in schoolchildren with asthma and allergic rhinitis. Pediatr Allergy Immu 2011;22:178-85.

46. Wakamatsu TH, Dogru M, Ayako I, Takano Y, Matsumoto Y, Ibrahim OM et al. Evaluation of lipid oxidative stress status and inflammation in atopic ocular surface disease. Mol Vis 2010;16:2465-75.

47. Fullard RJ, Snyder C. Protein levels in nonstimulated and stimulated tears of normal human subjects. Invest Ophth Vis Sci 1990;31(6):1119-26.

48. Zhou L, Wei R, Zhao P, Koh S K, Beuerman RW, Ding C. Proteomic analysis revealed the altered tear protein profile in a rabbit model of Sjogren's syndrome-associated dry eye. Proteomics 2013;13(16):2469-81.

49. Hegarty DM, David LL, Aicher SA. Lacrimal Gland Denervation Alters Tear Protein Composition and Impairs Ipsilateral Eye Closures and Corneal Nociception. Invest Ophth Vis Sci 2018;59(12):5217-24.

50. Leonardi A, Lazzarini D, Bortolotti M, Piliego F, Midena E, Fregona I. Corneal confocal microscopy in patients with vernal keratoconjunctivitis. Ophthalmology 2012;119(3):509-15.

51. Hu Y, Matsumoto Y, Adan ES, Dogru M, Fukagawa K, Tsubota K et al. Corneal in vivo confocal scanning laser microscopy in patients with atopic keratoconjunctivitis. Ophthalmology 2008;115(11):200412.

52. Leonardi A. Allergy and allergic mediators in tears. Exp Eye Res 2013;117:106-17. 

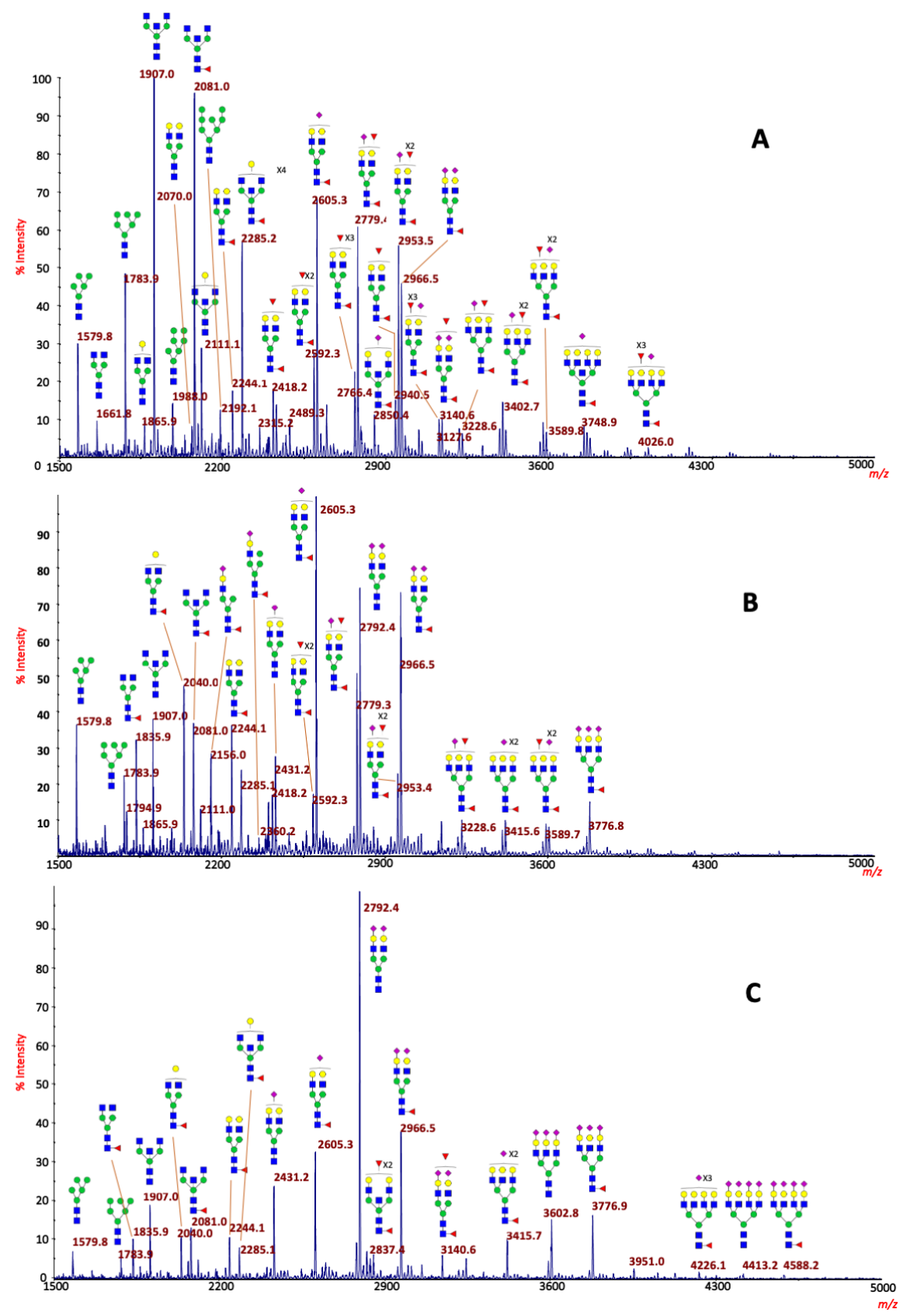

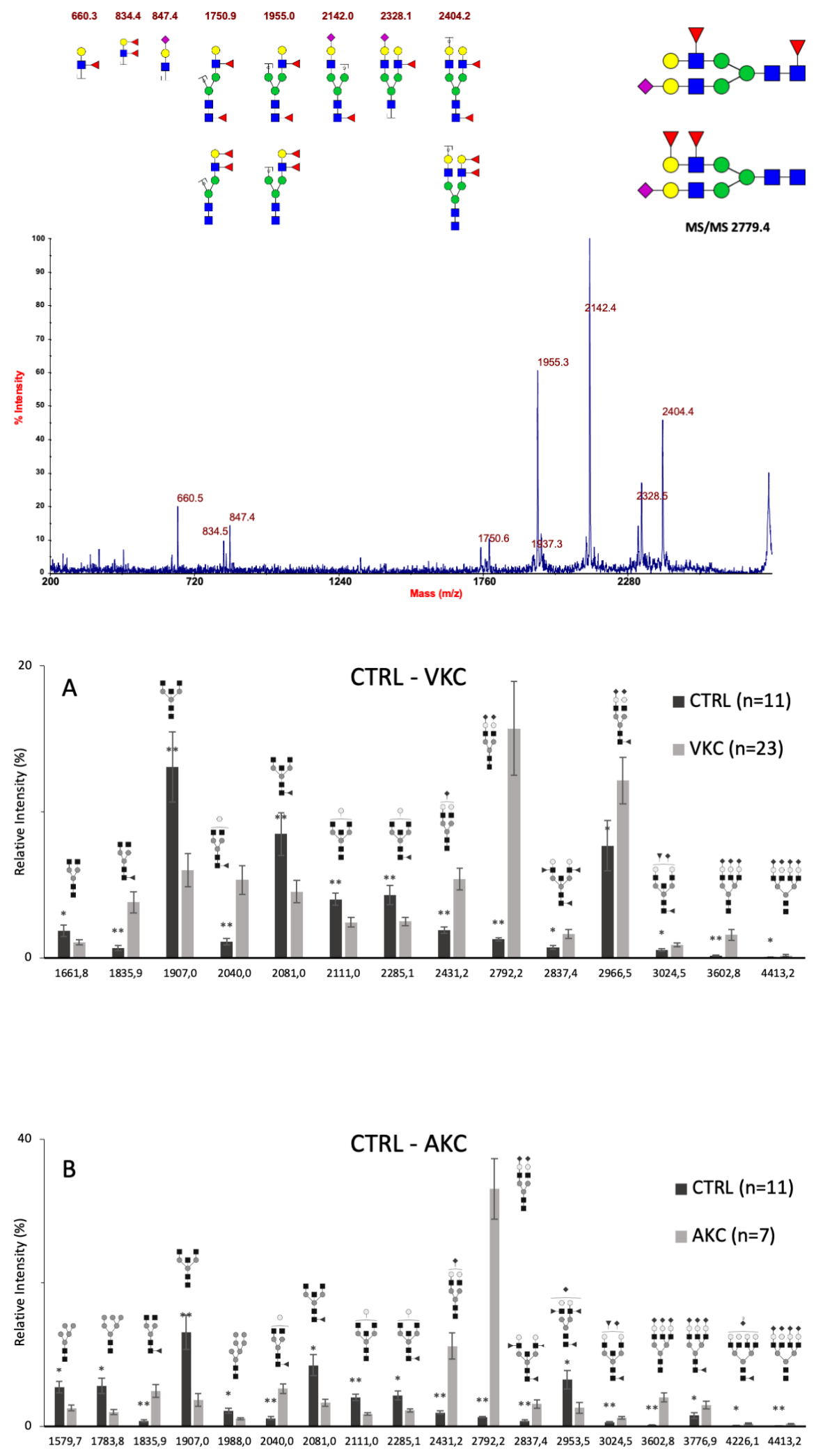


\section{Tear serotransferrin}

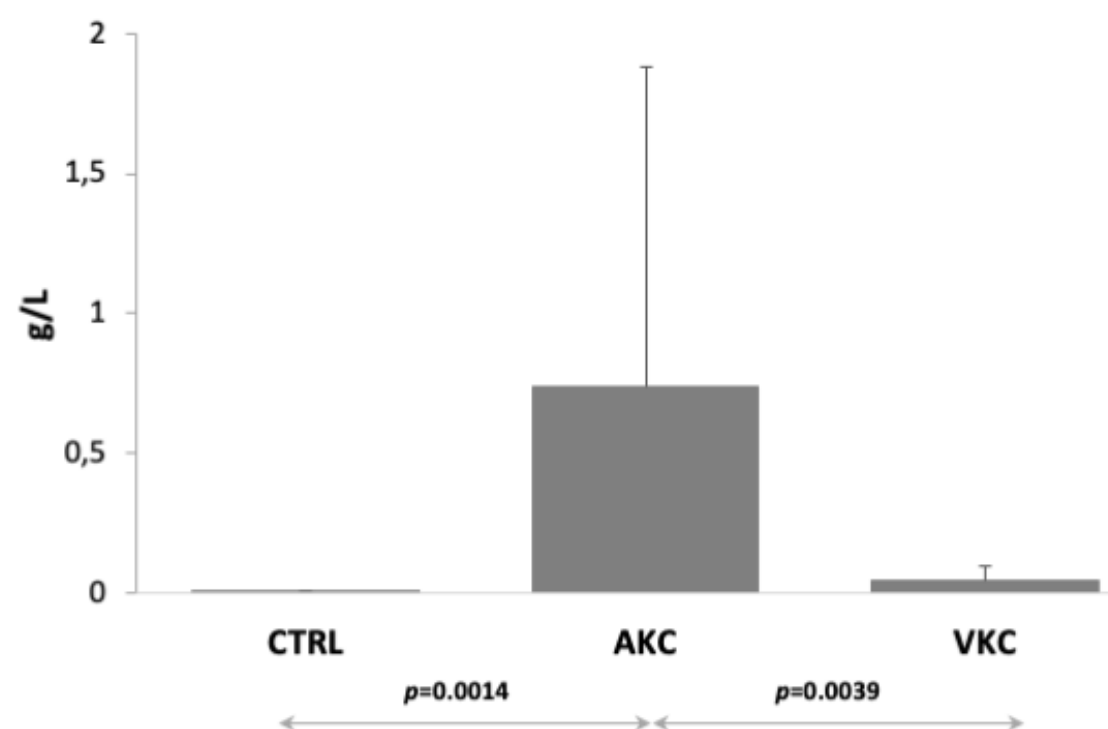

$p=0.0014$ 